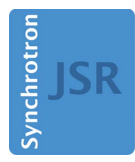

JOURNAL OF SYNCHROTRON RADIATION

ISSN 1600-5775

Received 1 July 2019

Accepted 17 October 2019

Edited by A. Stevenson, Australian Synchrotron, Australia

Keywords: beryllium; optical constants; refractive index; photoabsorption; near-edge X-ray absorption fine-structure spectroscopy; free-standing thin film.

Supporting information: this article has supporting information at journals.iucr.org/s

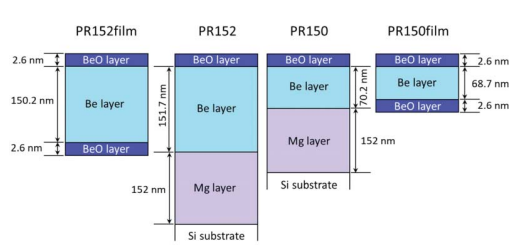

(C) 2020 International Union of Crystallography

\section{Optical constants of sputtered beryllium thin films determined from photoabsorption measurements in the spectral range $20.4-250 \mathrm{eV}$}

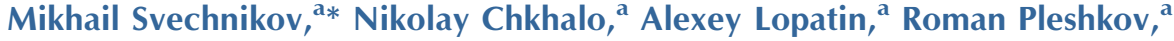

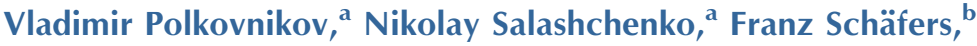 \\ Mewael G. Sertsu, ${ }^{\mathrm{b}}$ Andrey Sokolov ${ }^{\mathrm{c}}$ and Nikolay Tsybin ${ }^{\mathrm{a}}$
}

\footnotetext{
anstitute for Physics of Microstructures, Academicheskaya 7, Nizhny Novgorod 603087, Russian Federation, ${ }^{\mathbf{b}}$ Department of Nanooptics and Technology, Helmholtz-Zentrum Berlin, Albert-Einstein-Straße 15, D-12489 Berlin, Germany, and ${ }^{\mathbf{C}}$ Department Precision Gratings, Helmholtz-Zentrum Berlin, Albert-Einstein-Straße 15, D-12489 Berlin, Germany. ${ }^{*}$ Correspondence e-mail: svechnikovmv@gmail.com
}

In this work, the refractive index of beryllium in the photon energy range 20.4$250 \mathrm{eV}$ was experimentally determined. The initial data include measurements of the transmittance of two free-standing Be films with thicknesses of $70 \mathrm{~nm}$ and $152 \mathrm{~nm}$, as well as reflectometric measurements of similar films on a substrate. Measurements were carried out at the optics beamline of the BESSY II synchrotron radiation source. The absorption coefficient $\beta$ was found directly from the transmission coefficient of the films, and the real part of the polarizability $\delta$ was calculated from the Kramers-Kronig relations. A comparison is carried out with results obtained 20 years ago at the ALS synchrotron using a similar methodology.

\section{Introduction}

Beryllium is an important material in X-ray optics, with high transparency in several spectral ranges. It is widely used as a material for: windows of vacuum chambers, as it allows for the output of X-ray radiation; refractive lenses of synchrotron beamlines, as it allows for flexible control over beam focusing; and substrates for X-ray optical elements with high elasticity and thermal conductivity with a small mass (Beguiristain et al., 2002; Chkhalo, Mikhailenko et al., 2019; ESRF, undated). Be is one of the few transparent materials in the extreme-ultraviolet wavelength range (EUV) at energies below the $K$-absorption edge of $111.75 \mathrm{eV}$. For this reason (and also due to its relatively high melting point of $1278^{\circ} \mathrm{C}$ for the bulk material) it can be used in absorption filters (Chkhalo et al., 2016) and as a component of highly reflective multilayer mirrors for a number of spectral ranges: W/Be mirrors for $0.67-3.12 \mathrm{~nm}$ (Akhsakhalyan et al., 2019), Mo/Be for $11.4 \mathrm{~nm}$ (solar astronomy and lithography) (Bogachev et al., 2016; Montcalm et al., 1998; Skulina et al., 1995), Mo/Be/Si for $13.5 \mathrm{~nm}$ (solar astronomy and lithography) (Chkhalo, Gusev et al., 2017), Be/ $\mathrm{Al}$ for $17.1-19 \mathrm{~nm}$ and $30.4 \mathrm{~nm}$ and $\mathrm{Be} / \mathrm{Si} / \mathrm{Al}$ for $17.1-19 \mathrm{~nm}$ (solar astronomy) (Chkhalo, Pariev et al., 2017; Chkhalo, Lopatin et al., 2019), Ti/Be for $25 \mathrm{~nm}$ (Chkhalo et al., 1995), and $\mathrm{Be} / \mathrm{Mg}$ for $30.4 \mathrm{~nm}$ (solar astronomy) (Polkovnikov et al., 2019). Knowledge of the optical constants (the real and imaginary parts of the refractive index) is necessary to simulate the optical properties and to carry out reflectometric diagnostics of Be-containing optical elements. The most accurate experimental measurement of photoabsorption in 
thin Be films in the energy range $40-250 \mathrm{eV}$ was performed by Soufli et al. (1999); these data are available from the CXRO database of optical constants (http://henke.lbl.gov/optical_ constants/). Prior to this, measurements were performed in the vicinity of the Be absorption edge, 110-170 eV (Haensel et al., 1970), at individual spectral points (Barstow et al., 1983), while the remainder of the soft X-ray (SXR) and EUV ranges were described by calculations (Henke et al., 1993). However, the processing of experimental reflectometric curves often revealed significant discrepancies from simulation results using CXRO constants in the low-energy region, and in particular those describing Be/Mg mirrors at a wavelength of $30.4 \mathrm{~nm}(40.78 \mathrm{eV})$ (Polkovnikov et al., 2019). The reasons for the discrepancy may be incorrect assumptions about the structure and chemical composition of the contaminated surface layer, errors in the thicknesses and roughness of the internal layers, or the difference between real and tabulated optical constants. To exclude the last factor, we decided to measure the refractive index of $\mathrm{Be}$ in the EUV and SXR ranges, in a similar way to Soufli et al. (1999). Among other things, the availability of an alternative dataset obtained by a similar technique increases the reliability of the tabulated optical constants in the selected energy range.

In this paper, we followed the methodology developed in the works of Delmotte et al. (2018) and Soufli et al. (2019). The thicknesses of the films and oxide layers were determined by EUV reflectometry and checked by transmission modeling. The method of free-standing film transmission measurements over a wide range of spectral wavelengths was chosen to determine the optical constants, since in this case the interpretation of the measurement results does not depend on the quality of the interfaces of the films, and the effect of surface contamination (mainly oxidation) can be compensated in a very simple way.

\section{Experimental section}

\subsection{Deposition of Be films}

The Be films were deposited in a specially certified laboratory at IPM RAS, since Be is a highly toxic material. However, only Be dust constitutes a threat to human health, and its effects are cumulative; according to sanitary norms, storage, research and long-term operation with Be-containing films are not harmful to human health, and these activities do not require special precautions.

Deposition was carried out by direct-current magnetron sputtering at the facility, pumped out to a residual pressure of $4 \times 10^{-5} \mathrm{~Pa}$. The working pressure of argon during the deposition was $0.11 \mathrm{~Pa}$, and the chemical purity of Ar was about $99.99 \%$. The target materials were disks of diameter $150 \mathrm{~mm}$ and thickness $5 \mathrm{~mm}$. The chemical purity of the bulk Be target, according to specification, is $99 \%$ (in atomic concentration). Main impurities are: O, 0.7\%; C, $0.09 \%$; $\mathrm{Fe}, 0.04 \%$ (in atomic concentration). The process utilized $270 \mathrm{~W}$ of power for the Be target and $160 \mathrm{~W}$ for the Mg target.
The films were deposited on a Si wafer with a diameter of $100 \mathrm{~mm}$, with a microroughness of less than $0.2 \mathrm{~nm}$. Be was sputtered on top of a $152 \mathrm{~nm}$-thick magnesium sacrificial layer. After deposition, the substrate was divided into several parts. For the manufacture of free-standing films, the sacrificial $\mathrm{Mg}$ layer was dissolved in acetic acid, while for reflectometric measurements we used films on the substrate.

\subsection{EUV and SXR measurements}

Reflectivity and transmissivity measurements were carried out in the EUV and SXR regions using at-wavelength metrology equipment, with an 11-axis reflectometer endstation on the optics beamline of the BESSY II synchrotron radiation source (Schäfers et al., 2016; Sokolov et al., 2016). The reflectometer is located at the end of the optics beamline and is designed specifically for metrology of the optical elements in the SXR and EUV ranges at working wavelengths. The range of wavelengths available at this station is 0.62 $124 \mathrm{~nm}(10-2000 \mathrm{eV})$. The monochromatization of the beam was carried out with a SX-700 monochromator with a replaceable flat grating (which could be switched between two installed gratings of 1200 and 150 lines $\mathrm{mm}^{-1}$ ). The spectral resolution $E / \Delta E=2000-5000$, the setting accuracy of the wavelength was $0.02 \%$, and spectral impurities were less than $0.01 \%$. Polarization was variable, from linear to elliptical. The energy scale was calibrated along the edges of the absorption of thin-film filters and along the absorption lines of gasses. To suppress high orders, a four-mirror system operating in the field of total external reflection was used (a high-order suppressor or HiOS). The reflectometer was equipped with an 11-axis goniometer, with an angle setting accuracy of $0.02^{\circ}$. The size of the incident beam in this experiment was about $0.6 \mathrm{~mm} \times 0.25 \mathrm{~mm}$ (width $\times$ height). The active area of the detector of $4 \mathrm{~mm} \times 4 \mathrm{~mm}$ was sufficient to receive specular reflection and most of the scattered part of the reflected beam.

Measurements of the spectral and angular reflection curves from films on substrates were carried out with a grating of 1200 lines $\mathrm{mm}^{-1}$. The transmission coefficient of the films was measured in the energy range $14-630 \mathrm{eV}$, but only part of this range (20.4-250 eV) was used to extract optical constants; the reasons for this are discussed below. For measurements in the range $14-110 \mathrm{eV}$, a grating with 150 lines $\mathrm{mm}^{-1}$ was used and, for the range $36-630 \mathrm{eV}, 1200$ lines $\mathrm{mm}^{-1}$ was used. In the intersection area, the data were stitched. Calibration was performed at the absorption edges of the $\mathrm{Al}, \mathrm{Mg}, \mathrm{Si}, \mathrm{Be}, \mathrm{B}, \mathrm{Fe}$, $\mathrm{Ti}, \mathrm{Cr}$ and $\mathrm{Cu}$ thin-film filters. The HiOS mirrors used were uncoated bulk $\mathrm{Si}$ or had a coating of $\mathrm{C}$ or $\mathrm{AlF}_{3}$; the grazing angle for these was $3-40^{\circ}$, depending on the energy. Absorption filters were not used in the measurement process.

\section{Beryllium thin films}

The thickness of the Be films and the thickness of the $\mathrm{BeO}$ oxide layer, which forms on the surface in normal conditions, were determined from the results of measurements of the reflection and transmission coefficients. The reflection curves 


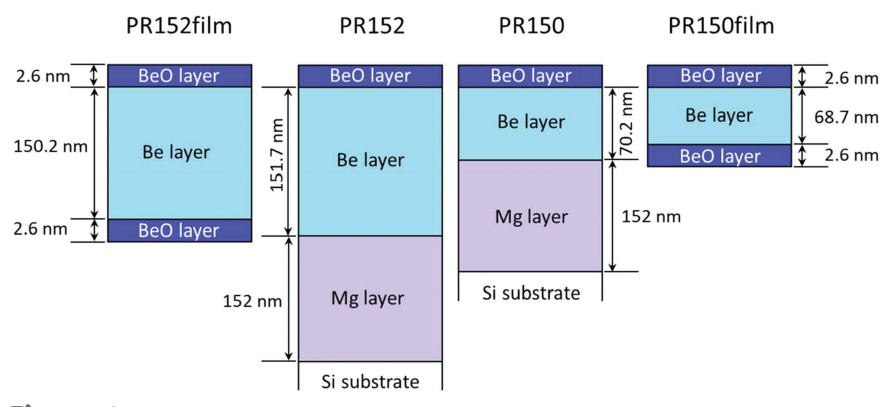

Figure 1

Schematic structure of samples on substrates and free-standing films. The $\mathrm{Mg}$ layer thickness shown here is nominal, and the thicknesses of $\mathrm{Be}$ and $\mathrm{BeO}$ are determined according to the reflectometry of the samples on the substrates and transmission measurements of free-standing films.

were measured for Be-on-Mg films on the substrate. Thus, the study involved four samples, which are schematically shown in Fig. 1. Samples marked PR150film and PR152film are freestanding films corresponding to the PR150 and PR152 samples after dissolving the $\mathrm{Mg}$ layer in an aqueous solution of acetic acid. After dissolving the Mg layer, the Be films were caught on frames with a through-hole of diameter $8 \mathrm{~mm}$. As revealed by subsequent measurements of the transmission spectra, no jumps were observed in the region of the $\mathrm{Mg} L_{2,3}$-absorption edges (49.5-49.9 eV), meaning that there was no residual $\mathrm{Mg}$ on the free-standing samples.

The thicknesses of the 'pure' Be core layer and the natural surface oxide $\mathrm{BeO}$ film were determined by reflectometric reconstruction. Based on the results of reflectometric modeling, it was found that the interlayer roughness in the structures on the substrate was about $4.5 \mathrm{~nm}$ (the root-meansquare value). This large value is apparently due to the
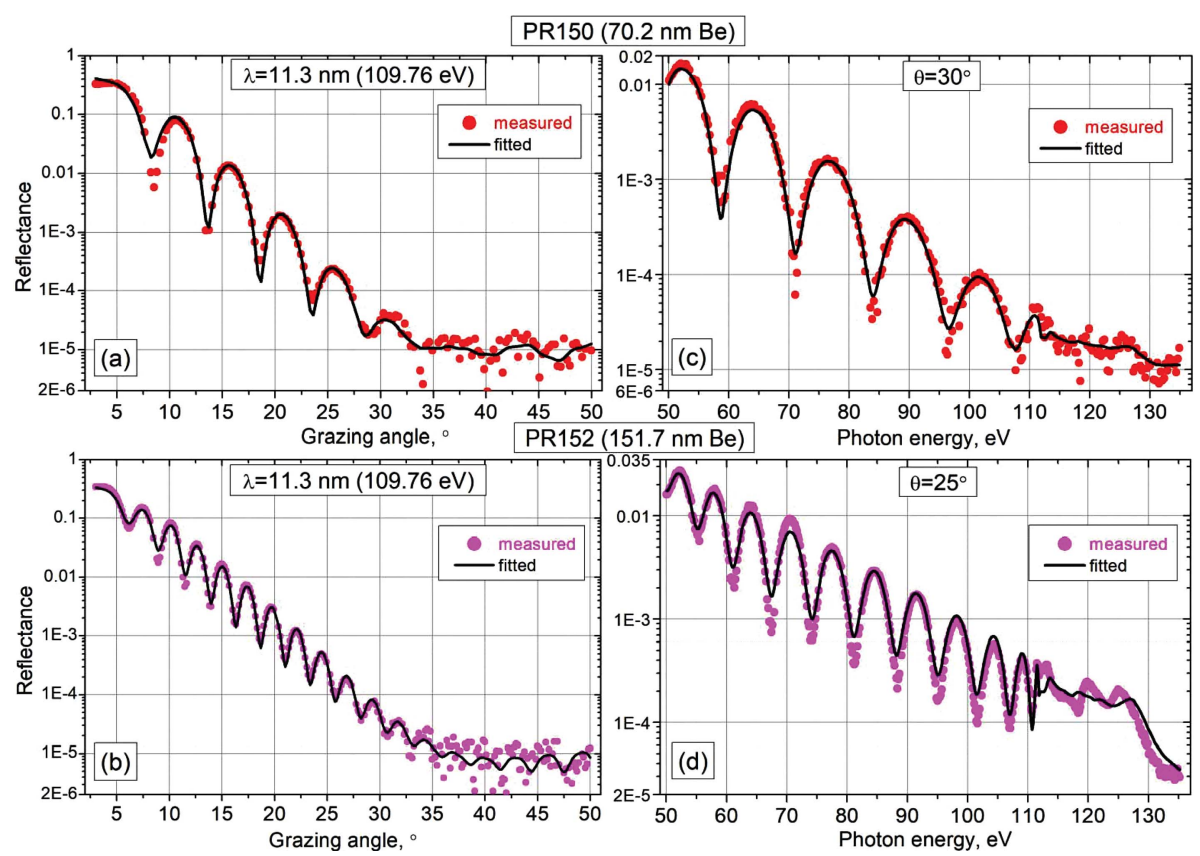

Figure 2

Angular and spectral reflection curves from films on substrates. In $(a)$ and $(b)$ the wavelength is fixed; in $(c)$ and $(d)$ the grazing angle of the probe beam is fixed. roughness growth in the thick 'sacrificial' layer of $\mathrm{Mg}$. The difference in the thicknesses of the Be layer for the samples on the substrate and the free-standing films is associated with the appearance of an oxide film on the reverse side. At nominal densities of $\rho(\mathrm{Be})=1.848 \mathrm{~g} \mathrm{~cm}^{-3}$ and $\rho(\mathrm{BeO})=3 \mathrm{~g} \mathrm{~cm}^{-3}$, the formation of $2.6 \mathrm{~nm} \mathrm{BeO}$ requires about $1.5 \mathrm{~nm}$ of 'pure' Be. Hence, the Be layer between two $\mathrm{BeO}$ layers in free-standing films is $1.5 \mathrm{~nm}$ thinner than the $\mathrm{Be}$ layer between $\mathrm{BeO}$ and $\mathrm{Mg}$ in the films on the substrate.

Figs. 2(a)-2(d) show the angular and spectral reflectivity curves for 'thick' (PR152) and 'thin' (PR150) films with the fitted model curves. The simulation was carried out using the Multifitting software program (our development, in preparation for publication). Both samples were reconstructed simultaneously, with the imposed condition that the thickness and density of the $\mathrm{BeO}$ oxide layer were the same for both films. Modeling the transmission curves of free-standing films (firstly in the region of the $K$-edge of oxygen) with the same oxide layer confirmed the consistency of the parameters used.

\section{Transmittance of Be thin films}

The transmission curves of free-standing PR150film and PR152film were measured in the range $14-630 \mathrm{eV}$, as shown in Fig. 3. However, due to the rapidly decreasing intensity of the probe beam towards the region of low energies and the increasing absorption of $\mathrm{Be}$, the dynamic range of the optics beamline endstation did not allow us to measure transmission below $\sim 10^{-3}$. For PR150film, the smallest energy value at which the signal noticeably exceeds the noise is $18.5 \mathrm{eV}$ at $T \simeq$ $4.5 \times 10^{-3}$, and, for PR152film, the lower bound is $20.4 \mathrm{eV}$ with $T \simeq 9 \times 10^{-4}$. The main features of the curves are the structures of the of Be $K$-, C $K$ - and O $K$-absorption edges. It is important to note that, in spite of the fact that $\mathrm{C}$ and $\mathrm{O}$ are present in the studied samples in certain quantities, they are also present on the surface of the optical elements of the synchrotron beamline. Since this spectrum is present in both the incident and transmitted beams, the spectrum of the beamline optics should be compensated after dividing the transmitted signal by the incident signal. A necessary condition for this is the monochromaticity of the incident and transmitted beam, i.e. low intensity of high orders and scattered radiation.

If the main diffraction order of the grating falls within the region of strong absorption, then, after the beam passes the polluted beamline optics, the degree of monochromatization drops sharply, and in practice the beam has significant spectral impurities. The resulting transmission spectrum of the sample in this area is correspondingly distorted. In our 

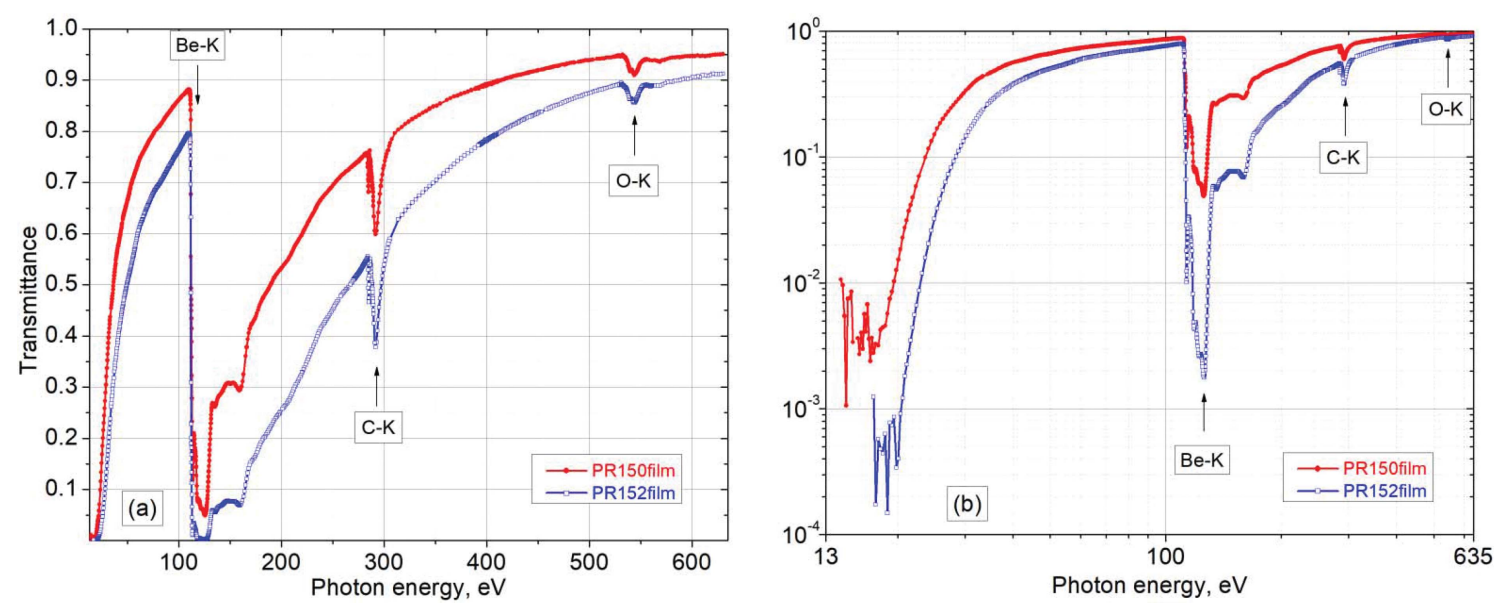

Figure 3

Transmission spectra of the samples on a $(a)$ linear and $(b)$ double logarithmic scale. In the low-energy region, the curves fall to the noise level due to a decrease in the radiation intensity.

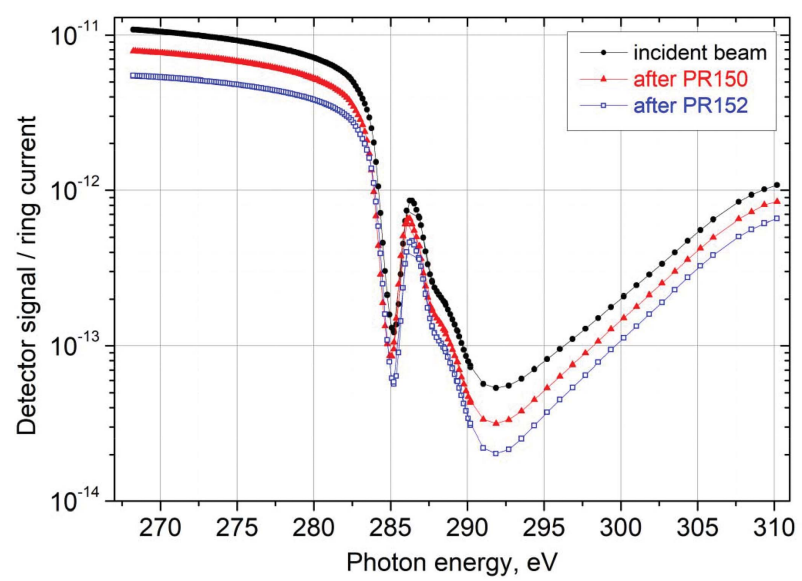

Figure 4

Reduction in the signal at the detector when measuring the incident and transmitted beams in the vicinity of the $\mathrm{C} K$-absorption edge.

case, strong suppression of the main order by the beamline optics occurs in the region of carbon absorption. Fig. 4 shows the signal from the detector when measuring the spectrum in the vicinity of the $\mathrm{C} K$-absorption edge. The intensity of the main diffraction order corresponding to the monochromated beam decreases by a factor of $\sim 10-200$ in the region 280 $310 \mathrm{eV}$ and exceeds the noise level by a factor of only $10-100$.

Fig. 5 shows the 'differential' spectrum, which corresponds to the amount of material (and its chemical composition) by which the two free-standing films differ. This spectrum is the ratio of the transmission spectra $T_{\mathrm{PR} 152} / T_{\mathrm{PR} 150}$. Since it is assumed that the surface layers containing the majority of the contamination are the same for both samples, this 'differential' film consists of 'internal' Be and has a thickness $d(\mathrm{PR} 152 \mathrm{film})-d(\mathrm{PR} 150 \mathrm{film})=150.2 \mathrm{~nm}-68.7 \mathrm{~nm}=$ $81.5 \mathrm{~nm}$. As can be seen from Fig. 5(a), compared with the spectra in Fig. 3(a) the edge of oxygen in the 'differential' spectrum has almost disappeared. This means that both the PR152film and PR150film samples contain a small total amount of oxygen throughout the entire thickness. Incomplete compensation may be associated with the difference in the amount of oxygen within the Be films of various thickness, and is also possible due to slight differences in the thickness of the surface oxides. Here we assume that the surface layers are fully identical, and model the 'differential' spectrum together with the individual spectra and reflectometric curves for both samples. Assuming that the main impurities are carbon and oxygen, the simulation showed that the chemical composition of the film thickness is $\mathrm{Be}_{1} \mathrm{C}_{x} \mathrm{O}_{y}$, where $x=0.012-0.020$ and $y=$ $0.003-0.006$, i.e. $1.2-2 \% \mathrm{C}$ and $0.3-0.6 \% \mathrm{O}$ (atomic concentrations). The best-fit values of $x$ and $y$ are: $x=0.015, y=0.004$, but $\mathrm{C}$ and $\mathrm{O}$ are intercorrelated in such a way that decreasing of $\mathrm{C}$ can be compensated by increasing of $\mathrm{O}$ and vice-versa. According to the specification of the Be target (where the stated atomic concentration contamination of $\mathrm{O}$ is $0.7 \%$ and of $\mathrm{C}$ is $0.09 \%$ ), we conclude that almost all of the carbon contamination came to the Be film from the residual atmosphere in the vacuum chamber. It is highly likely that the direct source of carbon was the rubber seals and polymeric thermal interface between target and cooling system. As for oxygen, there can be two main sources of film contamination: a contaminated target and residual water vapor in the chamber.

The previously mentioned distortion of the spectrum in the region $280-310 \mathrm{eV}$ leads to the observed absorption level in the region of the $\mathrm{C} K$-edge being higher than the real absorption associated with carbon impurities within the sample. Due to this effect, a strong C $K$-edge is still observed in the 'differential' spectrum, but transmittance modeling in the region $>310 \mathrm{eV}$ does not show a significant amount of carbon in the samples. Since the main goal of this work is to determine the optical constants in the lower-energy region, we used only the spectral region $<250 \mathrm{eV}$ for the reconstruction of the refractive index, as this region does not contain strong distortions such as an absorption structure artifact.

\section{Determination of Be optical constants}

The part of the 'differential' spectrum in the energy range 20.4-250 eV, as shown in Fig. 5, is the direct source of data 


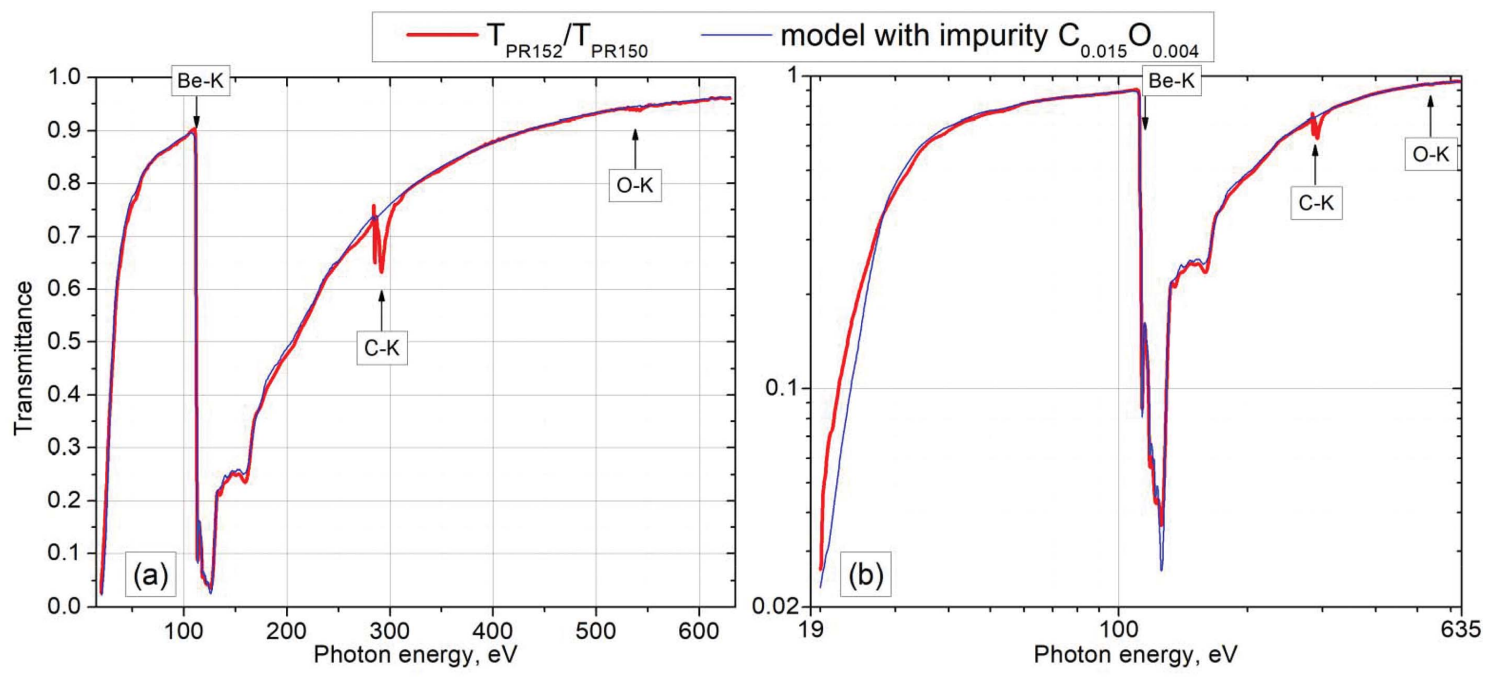

Figure 5

'Differential' transmittance spectrum on a $(a)$ linear and $(b)$ double logarithmic scale. Experimental spectrum (red curve) obtained by division of the PR152film measured transmission by the PR150film transmission and corresponds to the $81.5 \mathrm{~nm}$ Be film without surface contamination. Blue curve: modeled differential spectrum with impurity $\mathrm{C}_{0.015} \mathrm{O}_{0.004}$.

for the imaginary part of the refractive index. The difference spectrum is associated with the linear absorption coefficient of Be, $\alpha$, and the absorption of impurities, $\alpha_{x y}$,

$$
T(E, x)=\exp [-x \alpha(E)] \exp \left[-x \alpha_{\mathrm{C}_{x} \mathrm{O}_{y}}(E)\right]
$$

where $\alpha_{x y}$ is considered to be known. We obtained $\beta$ curves for best-fit values $x=0.015, y=0.004$, and a set of curves for
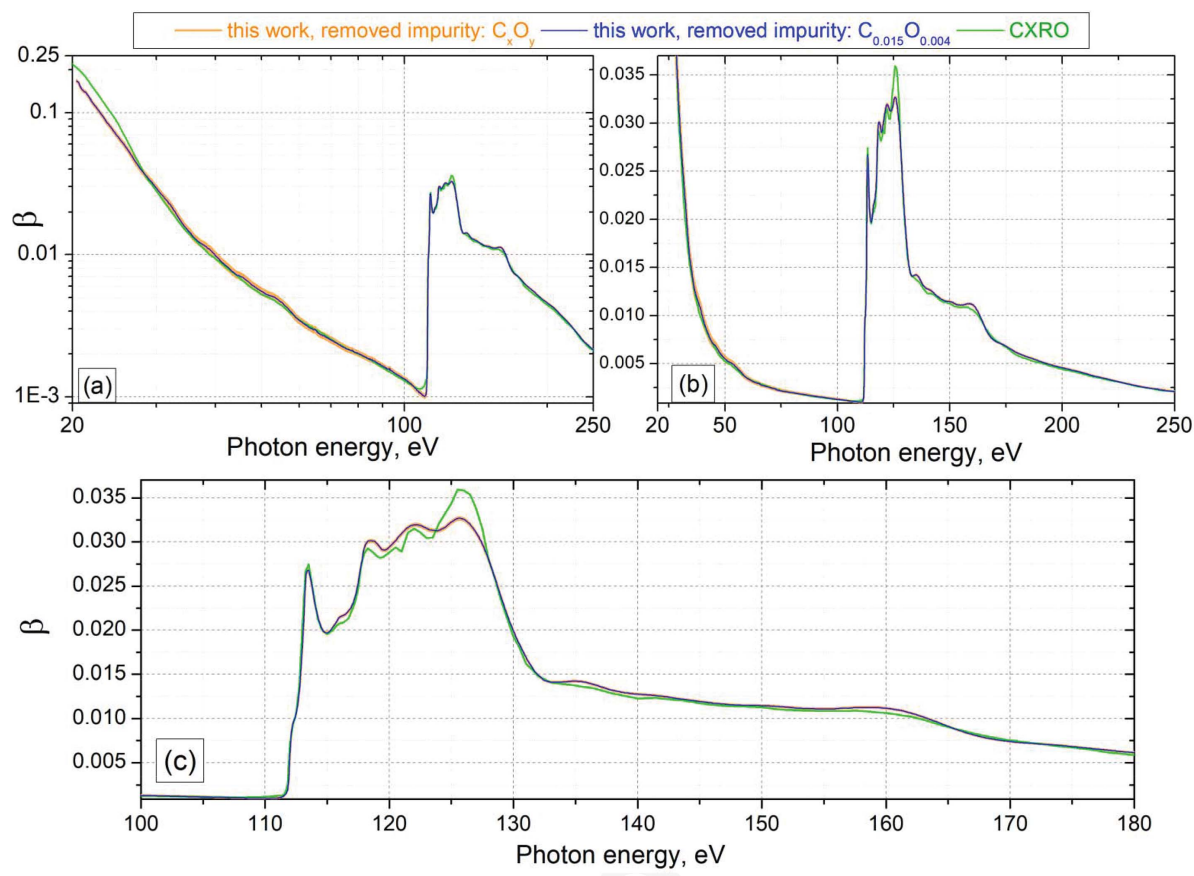

Figure 6

The absorption coefficient $\beta$ obtained from the spectrum in Fig. 5 in accordance with equation (1), and data from the CXRO database, on (a) a double logarithmic scale; $(b)$ a linear scale; $(c)$ a linear scale, zoomed beyond the absorption edge. The orange area shows the uncertainty (up to $\pm 5 \%$ ) associated with the $\mathrm{C}$ content in the range $1.2-2 \%$ and $\mathrm{O}$ in the range $0.3-0.6 \%$ (by atomic concentration). The blue line for $\mathrm{C}_{x=0.015} \mathrm{O}_{y=0.004}$ corresponds to the content of $1.5 \% \mathrm{C}$ and $0.4 \% \mathrm{O}$ inside the Be film. $x=0.012-0.020$ and $y=0.003-0.006$, to show the uncertainty. These data (for $\mathrm{C}$ and $\mathrm{O}$ absorption) are drawn from the CXRO optical constants database (imaginary parts are down to $10 \mathrm{eV}$ ). The thickness $x=81.5 \mathrm{~nm}$ is defined as the difference between the thicknesses of the studied samples, PR152film and PR150film. In equation (1), the beam is assumed to be monochromatic; when the high-order suppression system based on four mirrors is taken into account, the contributions of the second and higher diffraction orders can be considered negligible in the target range. The region in the vicinity of the $\mathrm{C}$ $K$-edge, where the monochromaticity condition is not satisfied due to contamination from the beamline optics and the strong absorption of the main diffraction order, was excluded from consideration. The refractive index $n=$ $1-\delta+i \beta$, where the imaginary part $\beta$ is proportional

to $\alpha$,

$$
\beta(E)=\lambda \alpha(E) / 4 \pi .
$$

Depending on the proportion of $\mathrm{C}$ and $\mathrm{O}$ in the film, an error bar for $\beta$ was obtained, as shown in Fig. 6 in orange. The uncertainty associated with impurities mainly affects the region up to the absorption edge of $\mathrm{Be}$, in the range 30$111 \mathrm{eV}$. As can be seen from the figure, all the main features of the absorption curve obtained in the work by Soufli et al. (1999) were also found in this study. The differences are solely quantitative: in the region of the beginning of the absorption edge, i.e. $105.5-111.5 \mathrm{eV}$, the 


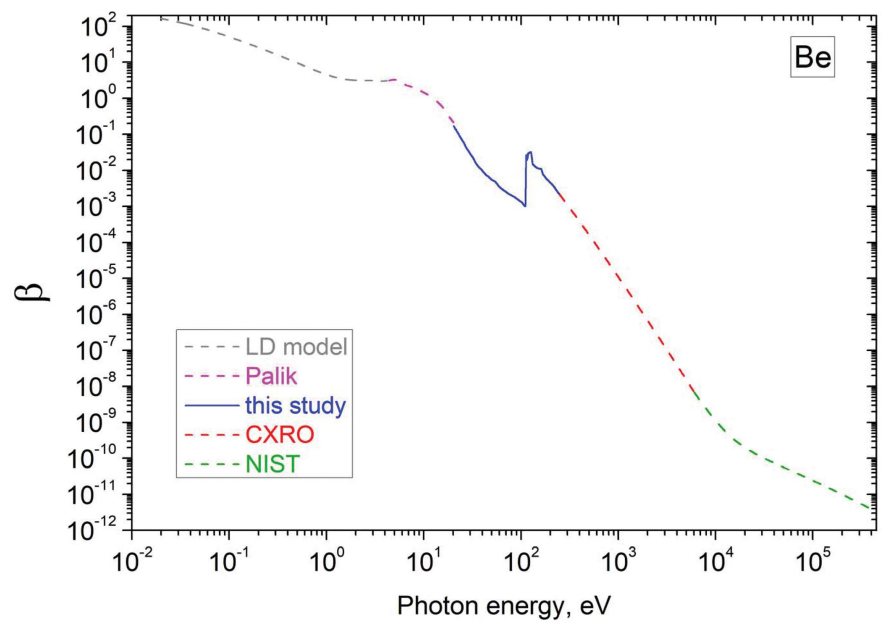

Figure 7

The total data set $\beta$ used to calculate $\delta$ with equation (3).

curve obtained in the present study has a much sharper appearance, the absorption peak at $123.8-127.5 \mathrm{eV}$ is less pronounced, and, in the range $20-28 \mathrm{eV}$, the values of $\beta$ found in the present work are $5-25 \%$ lower than those given in CXRO.

The Kramers-Kronig relations connecting the real and imaginary parts of the complex-valued function can be applied both to the dielectric permittivity and to the refractive index (Nussenzveig, 1972, pp. 17-19). Accordingly, the real part of the polarizability can be found using the equation

$$
\delta(E)=-\frac{2}{\pi} \int_{0}^{\infty} \frac{E^{\prime} \beta\left(E^{\prime}\right)}{E^{\prime 2}-E^{2}} \mathrm{~d} E^{\prime} .
$$

Finding $\delta$ in the range $20.4-250 \mathrm{eV}$ requires $\beta$ values for a wide range of energies $\left\{E_{\min }, E_{\max }\right\}, E_{\min } \ll 20.4 \mathrm{eV}, E_{\max } \gg$ $250 \mathrm{eV}$. The following datasets were used: in the range 0.02$4.2 \mathrm{eV}$, data were drawn from Rakić et al. (1998) and Polyanskiy (undated), calculated using the Lorentz-Drude model; for 4.2-20.4 eV, data were drawn from Palik (1991); for 20.4-
$250 \mathrm{eV}$, the data obtained in the present work were used; for $250-5950 \mathrm{eV}$, data from CXRO were used; and for 5950$432945 \mathrm{eV}$, data from Chantler et al. (undated) were used. These sources were also used by Delmotte et al. (2018) to obtain the optical constants for $\mathrm{Cr}$ and by Soufli et al. (2019) for Pt. The combined $\beta$ curve is shown in Fig. 7.

The result of applying the Kramers-Kronig relation is shown in Fig. 8. The difference in the experimentally determined curves for $\beta$ due to uncertainty arising from impurities does not have any noticeable effect on the value of $\delta$, and Fig. 8 therefore shows only one curve for the present study.

One of the criteria for the correctness of the found values of $\beta$ in a wide spectral range is the relation (Rodríguez-de Marcos et al., 2016)

$$
N_{\text {eff }}=\frac{4 m \varepsilon_{0}}{\pi n_{\mathrm{a}} \hbar^{2} e^{2}} \int_{0}^{\infty} E^{\prime} \beta\left(E^{\prime}\right) \mathrm{d} E^{\prime},
$$

where $N_{\text {eff }}=Z^{*}=Z-(Z / 82.5)^{2.37}$ is the effective number of electrons in an atom, $n_{\mathrm{a}}$ is the atomic density of the material, $m$ and $e$ are the mass and charge of an electron, respectively, $\varepsilon_{0}$ is the electric constant, and $h$ is Planck's constant. For Be, $Z=4$, and $Z^{*}=3.999$. The closer the value of $N_{\text {eff }}$ found by equation (4) to the value of $Z^{*}$, the more consistent the set of values of optical constants. This relation is called the 'sum rule'.

The effective number of electrons is calculated as $N_{\text {eff CXRO }}$ $=3.955$ using the CXRO data and $N_{\text {eff }}=3.928$ using the optical constants $\beta$ found in the present study in the range 20.4$250 \mathrm{eV}$. The limits of integration, as used in equation (3), were $0.02-433000 \mathrm{eV}$. The uncertainty of the absorption coefficient associated with the uncertainty of $\mathrm{C}$ and $\mathrm{O}$ impurities leads to values for $N_{\text {eff }}$ in the range 3.925-3.931. This variation is significantly smaller than the difference from $N_{\text {eff CXRO, and }}$ we can therefore assume that over the whole range of 20.4$250 \mathrm{eV}$ the CXRO data are more consistent with the combined wide-range $\beta$ set. However, we can find out which particular sub-range introduces the greatest error in the integral estimate. If, in the calculation, we take the value for $\beta$ found in the present work only for the region $28-250 \mathrm{eV}$, and
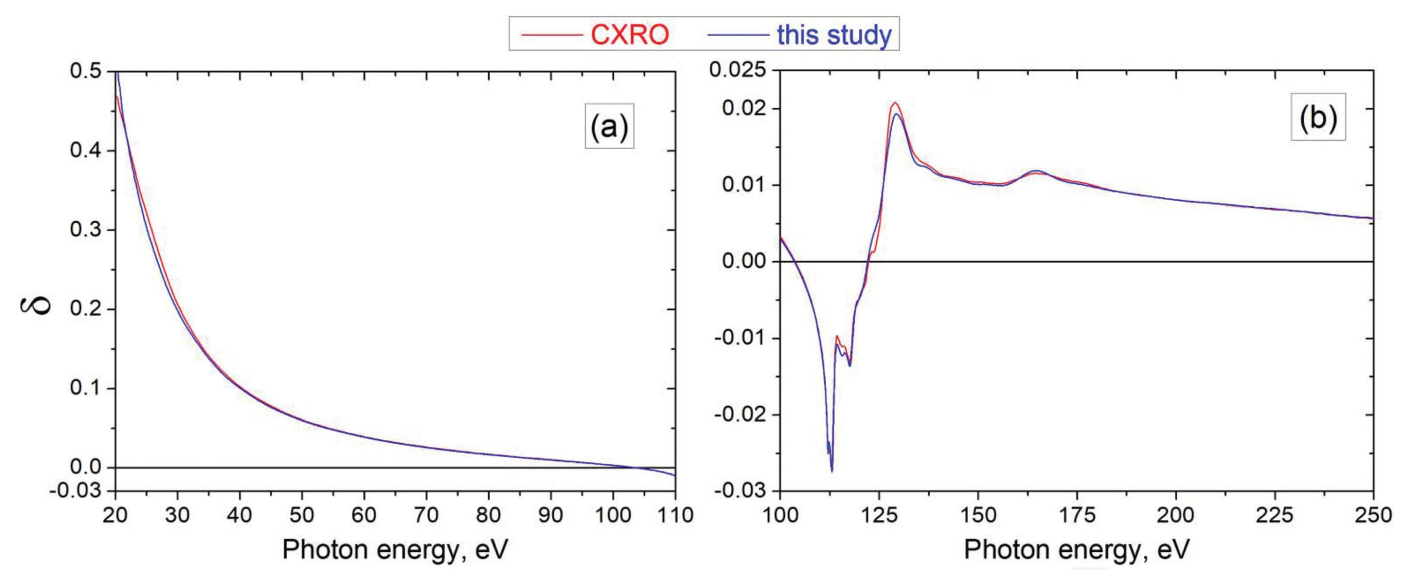

Figure 8

Polarizability $\delta$ as defined in this paper (blue curve) versus tabulated CXRO data (red curve); ( $a$ ) long-wavelength and (b) short-wavelength energy ranges are presented separately. 
use the CXRO data in the region 20.4-28 eV, then $N_{\text {eff }}=3.969$. This means that in the $28-250 \mathrm{eV}$ region the values we found turn out to be more consistent with the combined dataset (shown in Fig. 7) than the CXRO constants, but the values we found in the 20.4-28 eV range turn out to be less consistent. From Fig. 6(a), the difference between our data and the CXRO data can also be seen, and this increases with a decrease in photon energy. Thus, according to the 'sum rule', our data in the region 20.4-28 eV should be considered less reliable, and the values from CXRO should be preferred. There are also technical reasons for this that are associated with a sharp decrease in the intensity of the monochromatic beam when using a diffraction grating with 150 lines $\mathrm{mm}^{-1}$ in the low-energy region. In addition to the low dynamic range (about three orders of magnitude), there is a distortion of the spectrum and an increase in the proportion of radiation with shorter wavelengths; this has higher transmission through the sample, which in turn leads to an underestimate of the value of $\beta$.

\section{Conclusion}

In this paper, the optical constants of Be were determined by measuring the photoabsorption of free-standing films. In addition to oxidized and contaminated surface layers, $\mathrm{C}$ impurities of $1.2-2 \%$ and $\mathrm{O}$ impurities of $0.3-0.6 \%$ (in atomic concentration) were found in the sample. Two free-standing films with thicknesses $70 \mathrm{~nm}$ and $152 \mathrm{~nm}$ were studied for transmission and two corresponding films (with $\mathrm{Mg}$ sacrificial layers) on a substrate for reflection. Combining the obtained value for the absorption $\beta$ with data from the literature in the range $0.02-433000 \mathrm{eV}$ made it possible to find $\delta$ from the Kramers-Kronig relation, and also to check the obtained set of constants for consistency with the combined set based on the 'sum rule'. Thus, the main result of this study is independent confirmation of the reliability of the optical Be constants given in the CXRO database in the photon energy range $28-250 \mathrm{eV}$, up to the fine structure in the $\mathrm{Be} K$-absorption edge. The fine structure of the optical constants found here is generally consistent with the CXRO data, but there are quantitative differences. A test for the fulfillment of the 'sum rule' showed that at energies of $28-250 \mathrm{eV}$ the values obtained in this work are more consistent, while at energies of 20.4-28 eV the CXRO data are more consistent. This appears to be due to insufficient efficiency of the diffraction grating used in the experiment in the long-wavelength part of the spectrum, resulting in a strong drop in the intensity of the probe beam.

\section{Funding information}

The following funding is acknowledged: Russian Foundation for Basic Research (RFBR) (grant No. 19-07-00173; grant No. 19-02-00081; grant No. 18-02-00588; grant No. 17-52-150006; grant No. 18-42-520007; grant No. 18-32-00671; grant No. 18-
32-00173); State Assignment for the Institute for Physics of Microstructures, Russian Academy of Sciences (contract No. 0035-2014-0204).

\section{References}

Akhsakhalyan, A. A., Vainer, Y. A., Garakhin, S. A., Elina, K. A., Zavertkin, P. S., Zuev, S. Y., Ivlyushkin, D. V., Nechay, A. N., Nikolenko, A. D., Pariev, D. E., Pleshkov, R. S., Polkovnikov, V. N., Salashchenko, N. N., Svechnikov, M. V. \& Chkhalo, N. I. (2019). J. Surf. Investig. 13, 1-7.

Barstow, M. A., Lewis, M. \& Petre, R. (1983). J. Opt. Soc. Am. 73, 1220.

Beguiristain, H. R., Cremer, J. T., Piestrup, M. A., Gary, C. K. \& Pantell, R. H. (2002). Opt. Lett. 27, 778-780.

Bogachev, S. A., Chkhalo, N. I., Kuzin, S. V., Pariev, D. E., Polkovnikov, V. N., Salashchenko, N. N., Shestov, S. V. \& Zuev, S. Y. (2016). Appl. Opt. 55, 2126-2135.

Chantler, C. T., Olsen, K., Dragoset, R. A., Chang, J., Kishore, A. R., Kotochigova, S. A. \& Zucker, D. S. (undated). X-ray Form Factor, Attenuation, and Scattering Tables, NIST Standard Reference Database 66, http://physics.nist.gov/ffast.

Chkhalo, N. I., Drozdov, M. N., Kluenkov, E. B., Kuzin, S. V., Lopatin, A. Y., Luchin, V. I., Salashchenko, N. N., Tsybin, N. N. \& Zuev, S. Y. (2016). Appl. Opt. 55, 4683-4690.

Chkhalo, N. I., Fedorchenko, M. V., Kovalenko, N. V., Kruglyakov, E. P., Volokhov, A. I., Chernov, V. A. \& Mytnichenko, S. V. (1995). Nucl. Instrum. Methods Phys. Res. A, 359, 121-126.

Chkhalo, N. I., Gusev, S. A., Nechay, A. N., Pariev, D. E., Polkovnikov, V. N., Salashchenko, N. N., Schäfers, F., Sertsu, M. G., Sokolov, A., Svechnikov, M. V. \& Tatarsky, D. A. (2017). Opt. Lett. 42, 50705073 .

Chkhalo, N. I., Lopatin, A. Y., Nechay, A. N., Pariev, D. E., Pestov, A. E., Polkovnikov, V. N., Salashchenko, N. N., Schäfers, F., Sertsu, M. G., Sokolov, A. A., Svechnikov, M. V., Tsybin, N. N. \& Zuev, S. Y. (2019). J. Nanosci. Nanotechnol. 19, 546-553.

Chkhalo, N. I., Mikhailenko, M. S., Pestov, A. E., Polkovnikov, V. N., Zorina, M. V., Zuev, S. Y., Kazakov, D. S., Milkov, A. V., Strulya, I. L., Filichkina, V. A. \& Kozlov, A. S. (2019). Appl. Opt. 58, 36523658.

Chkhalo, N. I., Pariev, D. E., Polkovnikov, V. N., Salashchenko, N. N., Shaposhnikov, R. A., Stroulea, I. L., Svechnikov, M. V., Vainer, Y. A. \& Zuev, S. Y. (2017). Thin Solid Films, 631, 106-111.

Delmotte, F., Meyer-Ilse, J., Salmassi, F., Soufli, R., Burcklen, C., Rebellato, J., JéRome, A., Vickridge, I., Briand, E. \& Gullikson, E. (2018). J. Appl. Phys. 124, 035107.

ESRF (undated). Focusing compound refractive beryllium lenses, http://www.esrf.eu/UsersAndScience/Experiments/DynExtrCond/ ID28/BeamlineLayout/OH2/FocusBeCRL.

Haensel, R., Keitel, G., Sonntag, B., Kunz, C. \& Schreiber, P. (1970). Phys. Status Solidi A, 2, 85-90.

Henke, B. L., Gullikson, E. M. \& Davis, J. C. (1993). At. Data Nucl. Data Tables, 54, 181-342.

Montcalm, C., Bajt, S., Mirkarimi, P. B., Spiller, E. A., Weber, F. J. \& Folta, J. (1998). Proc. SPIE, 3331, 42.

Nussenzveig, H. M. (1972). Causality and Dispersion Relations. New York, USA: Academic Press.

Palik, E. D. (1991). Handbook of Optical Constants of Solids II. Boston: Academic Press.

Polkovnikov, V. N., Chkhalo, N. I., Pleshkov, R. S., Salashchenko, N. N., Schäfers, F., Sertsu, M. G., Sokolov, A., Svechnikov, M. V. \& Zuev, S. Y. (2019). Opt. Lett. 44, 263-266.

Polyanskiy, M. N. (undated). Refractive index database, https:// refractiveindex.info.

Rakić, A. D., Djurišić, A. B., Elazar, J. M. \& Majewski, M. L. (1998). Appl. Opt. 37, 5271. 
Rodríguez-de Marcos, L. V., Méndez, J. A. \& Larruquert, J. I. (2016). J. Opt. 18, 075606.

Schäfers, F., Bischoff, P., Eggenstein, F., Erko, A., Gaupp, A., Künstner, S., Mast, M., Schmidt, J.-S., Senf, F., Siewert, F., Sokolov, A. \& Zeschke, T. (2016). J. Synchrotron Rad. 23, 67-77.

Skulina, K. M., Alford, C. S., Bionta, R. M., Makowiecki, D. M., Gullikson, E. M., Soufli, R., Kortright, J. B. \& Underwood, J. H. (1995). Appl. Opt. 34, 3727-3730.
Sokolov, A., Bischoff, P., Eggenstein, F., Erko, A., Gaupp, A., Künstner, S., Mast, M., Schmidt, J.-S., Senf, F., Siewert, F., Zeschke, T. \& Schäfers, F. (2016). Rev. Sci. Instrum. 87, 052005.

Soufli, R., Bajt, S. \& Gullikson, E. M. (1999). Proc. SPIE, 3767, 251258.

Soufli, R., Delmotte, F., Meyer-Ilse, J., Salmassi, F., Brejnholt, N., Massahi, S., Girou, D., Christensen, F. \& Gullikson, E. M. (2019). J. Appl. Phys. 125, 085106. 